\title{
Designing AM PDU and Analyzing Protocol for GEO Satellite LTE
}

\author{
Xu Xiaoling ${ }^{1, a,{ }^{*}}$, Cui Weijia ${ }^{2, b}$, Zhang Yankui ${ }^{3, c}$ \\ 1,2,3 The PLA Information Engineering University, Zhengzhou City, 450001, China \\ axu014xiaoling@163.com, bbwdm123 @aliyun.com, cwirelessmancs@163.com, *corresponding \\ author
}

Keywords: GEO satellite, LTE, AM PDU, Protocol Analysis

\begin{abstract}
Considering the urgency of integrated development between the satellite and ground, the integration of terrestrial LTE technology and satellite becomes a research hotspot. The transmission delay for GEO satellite communication is long, the SN length of existing AM PDU can't meet the requirements of satellite communications, for the reason, the SN length should be extended. Taking into account the increase of the bandwidth of the satellite link in the future, here number of bits of $\mathrm{SN}$ is extended to 18 , and the effective bits are $12 \mathrm{bits}$. On the basis of the design and project topics, we build protocol analysis equipment, focusing on analyzing signal interaction based on UU port between the user and eNB, theoretical reasoning and manually resolve prove the rationality of the design for platform, which is of great significance for promoting further development of satellite LTE technology.
\end{abstract}

\section{Introduction}

The ground LTE mobile communication system being applied to satellite communications system need adaptive transform, which becomes a research hot spot in the communication field ${ }^{[1][2]}$.GEO communication distance is remote, which can be relayed to more than 10,000 kilometers of wide range of communication, but has disadvantages of long transmission delay, the transmission time is 230 270ms between GEO satellite and terrestrial. Voice communications between the station must be approved by two-hop link in the satellite system based on the central station, so the transmission delay is up to $540 \mathrm{~ms}^{[3]}$. This paper analyzes adaptability of the LTE RLC AM mode in long transmission delay satellite links, according to adaptive research for satellite RLC UM mode proposed by Dou Zhi-bin who works at China Electronics Technology Group Corporation 54th Research Institute, the idea of the article is to set the range of sequence numbers according to the bandwidth delay product. This paper analyzes the range of parameter sn-Field Length in RLC AM and relies on the national 863 key project "Mobile Satellite Communication System Full physical validation equipment study". We simulate the whole process of GEO satellite communication in the ground, joining the protocol analysis equipment. Protocol analysis server connects eNB equipment via the Ethernet interface, a copy of downlink and uplink signaling data in the MAC layer based on UU interface are sent to protocol analysis server, which parses air interface signaling related to the user establishment process in the MAC, RLC, PDCP, and RRC each sublayer, and providing resource allocation parameters. Checking parameter with eNB, we can confirm whether interaction signaling meets the requirements of the standard protocol between eNB and UE equipment or not.

\section{Design For AM PDU}

The bandwidth delay product is determined the size of the SN mentioned by literature ${ }^{[4]}$, that is the sn-Field Length must satisfy the formula (1), wherein Blink is the link bandwidth after being removed overhead in the PHY and MAC layer (unit: bps), De2e is end delay between links (unit: s), $L$ is the length of RLC AMD PDU (unit: bit). Considering the GEO satellite communication features, if we take L $=200$ Byte, Blink $=24 M b p s, D_{e 2 e}=270 m s$ the bit numbers of sn-Field Length is not less than 12bits. Therefore GEO satellites requires a larger window, here the size of SN should be extended, in addition, taking into account the link bandwidth may be greater in the future, 
adding a byte for the header of the AMD PDU, SN would be extended from the 10-bit to 18 bits, with 12 effective bits. the other field represents the same meaning as in LTE. The structure of AMD PDU(no LI) show in Fig. $1^{[5]}$.

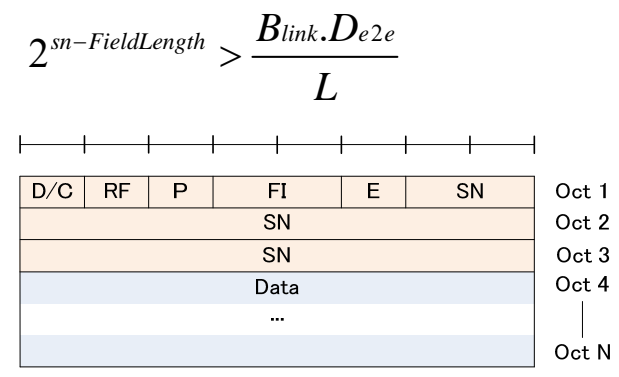

Fig. 1 AMD PDU (No LI)

\section{Protocol analysis test environment}

Specific experimental environment shows in Fig.2, the organic combination of various hardware and software simulate satellite communication in which LTE protocol analysis module parses interaction signaling for UU interface, in order to the aim of testing and validating interaction between eNB and UE whether meets the 3GPP standard protocols or not ${ }^{[6]}$. LTE protocol analysis module uses Microsoft Visual Studio 2010 compiler using C, C \#language in Windows server 2008 R2 enterprise server operating system.

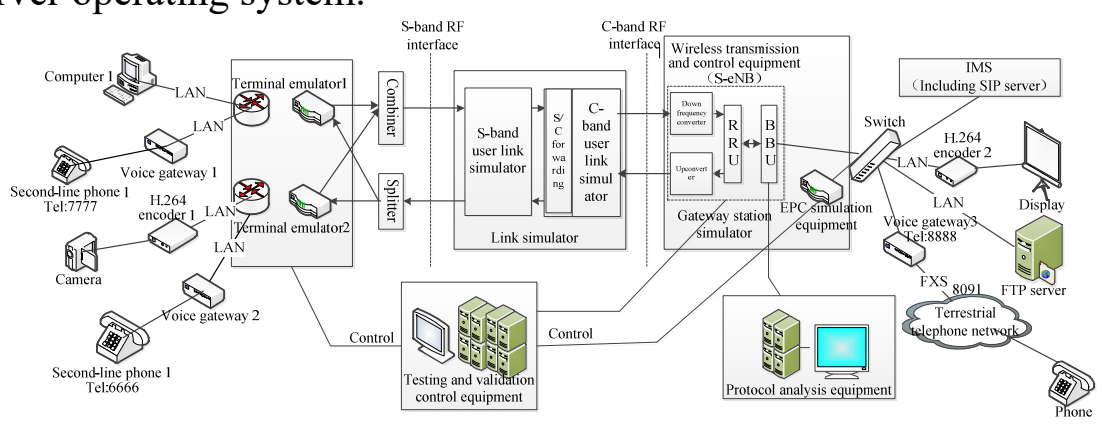

Fig. 2 Experimental environment

Software Architecture for Protocol Analysis. Due to the different message interface structure between downlink and uplink, we design downlink and uplink protocol analysis software architecture for the purpose of parsing protocol. Next, we show a detailed description of both design ideas.

The encapsulation of different messages each sub-layer through different physical channels, transport channels, logical channel is not necessarily the same. Therefore, considering that the protocol analysis module is mainly used to implement the signaling analysis in control plane, analysis is divided into three different branches to handle for the downlink message, as shown in Fig. 3. After receiving a frame of data, firstly parses data from underlying interface to obtain the value of C-RNTI. According to Table 7.1-1: RNTI values in 3GPP TS 36.321, we know that when the value is 65534 , the message is the paging message, which is carried by the transmission channel and logical channel of the PCH-PCCH; when the value is 65535 , the message is the system information, which is carried by the transmission channel and logical channel of the DLSCH-BCCH; under provision of the lower layer interface, when $\mathrm{PBCH}$ is non-empty, the message is master information block, that is the broadcast message. These three types of situations need not be processed by MAC, RLC, PDCP sublayer, after parsing from lower layer interface, call directly the top-level RRC functions to complete the signaling resolution. After calling the MAC_Decoding functions, if its LCID value is 0 , it can be determined that it belongs to DLSCH-CCCH channel. According to the 3GPP standard, its RLC layer belongs to the TM mode, so after processing in the MAC layer should be submitted to function of RRC ${ }^{[7]}$. Otherwise, it should be submitted to AM_RLC_Decoding function, and in turn by the PDCP_Decoding Functions, RRC layer function to obtain key parameters for the header parsing. 


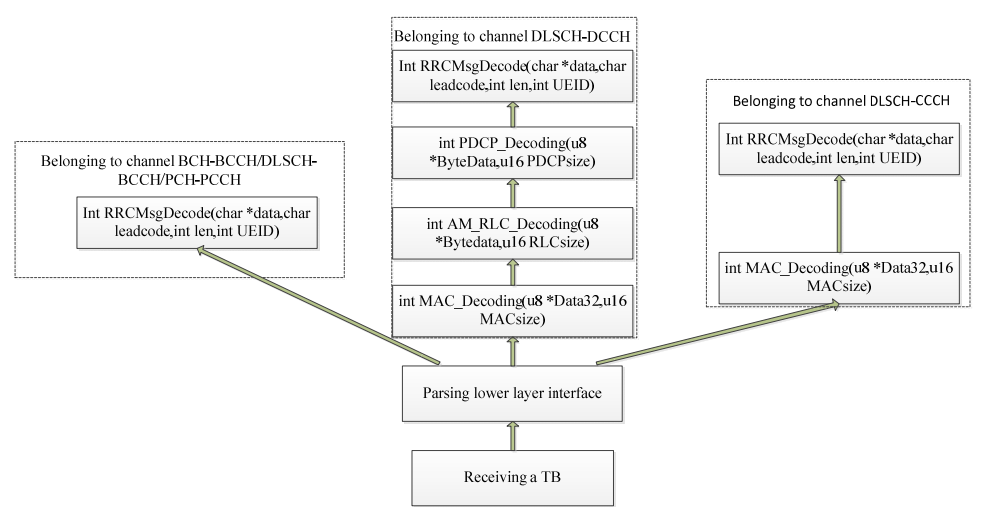

Fig. 3 downlink protocol analysis software architecture

Wherein function MAC_Decoding (u8 * Data U32, u16 MAXsize) is used for stripping header to get the message payload in MAC layer. the input parameter is the message payload pointer and byte numbers of a MAC PDU, the function will call RLC layer function when meet some conditions. Function AM_RLC_Decoding (u8 * Bytedata, u16 RLCsize) is used for parsing the RLC AM PDU in order to get AM SDU, the input parameter is message payload pointer of AM PDU data and byte numbers of RLC PDU, the function will call PDCP layer function when meet some conditions. Function PDCP_Decoding ( $\mathrm{u} 8 *$ ByteData, u16 PDCPsize) achieves stripping header for PDCP layer. Function RRCMsgDecode (char * data, char leadcode, int len, int UEID) is used to resolve the RRC layer data, the input parameters is the RRC PDU data pointer, leadcode represents the types of channel obtained from lower layer, value len is the number bytes of RRC PDU, value UEID is the C-RNTI value previously mentioned.

The channel types of uplink message are less, therefore, with the process of low-level interface, messages belongs to channel ULSCH-DCCH and ULSCH-CCCH. If the message belongs to channel ULSCH-CCCH, whose transmission is the transparent mode in the RLC layer, so after process in the MAC layer would be submitted to processing resolution in RRC layer. The ULSCH-DCCH message is subject to process of MAC, RLC, PDCP, RRC each sub-layer, as shown in Fig. 4.

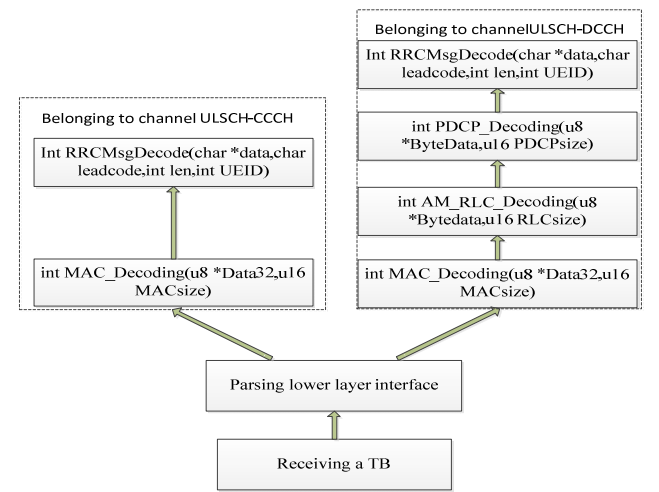

Fig. 4 uplink protocol analysis software architecture

Difficulties of Protocol Analysis. Packet parsing process involves collage of data package in RLC layer, if a big size data needs to be transmitted, we may need to send data several times. At this time, the receiver needs to combine many data segments successfully. If assumed that the data transmitted by several times are successfully received, without the loss of data, then the combination of package is a simple process, just joining few frames together, which is an ideal process. In the actual communication process, the same segment may be received multiple times, then just cache firstly received segment, later received segmented directly discarded until a new segment arrives. Of course, there is also the case of a section fails to successfully received, then wait receiving this segment, unless it receives new complete RLC SDU, the new data overwrites directly the segmented data packets in cache space. If the sequence number of the two data segments is determined as discontinuous, discards the data segment, without any process.

The design process relating to collage of data package in the Procedures: 
1) determining the frames before and after belong to the same user or not, because of the complexity in actual communication, the same time period may have many users random accessing to network;

2) If they belong to the same user, to further determine serial number ( $\mathrm{SN}$ ) of two frames are continuous, otherwise exit the program;

If two frames before and after belong to the same users and the serial number is continuous, and finally determine whether meets the requirements of combination of the package or not. That is behind the first segment of the RLC SDU only allows a combination of intermediate section or the last segment, the middle segment of the RLC SDU may be combined only the last segment, Finally, completing the combination of data packets.

\section{Test Results}

Protocol analysis software designed for GEO satellite LTE focus on the extension of SN in AM PDU and problem of collage of data package. Therefore, further analyzing protocol analysis software, and parsing data collected from the underlying interface to verify its design is correct or not. Due to the limited length of the article, where only include four groups of sample of the analysis results.

1) Sample one:

$00020038019100010000000000250000 \mathrm{ffff} 000000801 \mathrm{~d} 48381 \mathrm{e} 40 \mathrm{a} 0 \mathrm{e} 034868 \mathrm{a} 8240410 \mathrm{a} 00 \mathrm{c} 4 \mathrm{ba} 6 \mathrm{~d}$ 7e8a92dacce51c182843a45e91fde00000000000

Analytical results from underlying interface:

00801d48381e40a0e034868a8240410a00c4ba6d7e8a92dacce51c182843a45e91fde00000

After analysis, the message is carried by DLSCH-BCCH channel, the analytical results from underlying interface is shown in the above. Analysis results in RRC layer occupy big space, here is no longer displayed, manually processes verify analytical results are correct. The message is SIB2, contains all the public UE radio resource configuration information, using transparent mode in RLC layer.

2) Sample two:

0002006c001400010000000000590000003d0000212e1 fa000000801203a900089119a22ab33bc4 4cd55de66ef77f800879cfe0bd72a6c00074b6b749015581e580000000000000000000000000000000 000000000000000000000000000000000000000000000000000000000000000

Analytical results from underlying interface:

212e1fa000000801203a900089119a22ab33bc44cd55de66ef77f800879cfe0bd72a6c00074b6b74

$9015581 \mathrm{e} 580000000000000000000000000000000000000000000000000000000000000000000000$ 000000000000000000

Analytical results in MAC layer:

a000000801203a900089119a22ab33bc44cd55de66ef77f800879cfe0bd72a6c00074b6b74901558 $1 \mathrm{e} 5800000000$

Analytical results in RLC layer:

000801203a900089119a22ab33bc44cd55de66ef77f800879cfe0bd72a6c00074b6b749015581e58 00000000

Analytical results in PDCP layer:

0801203a900089119a22ab33bc44cd55de66ef77f800879cfe0bd72a6c00074b6b749015581e58

Analytical results in RRC layer:

\{

message c1: dlInformationTransfer: \{

rrc-TransactionIdentifier $\quad 0$,

criticalExtensions c1: dlInformationTransfer-r8: \{ dedicatedInfoType

dedicatedInfoNAS:

'075200112233445566778899AABBCCDDEEFF0010F39FC17AE54D8000E96D6E9202AB03CB

'H 
Manually processes verify analytical results are correct. The message is carried by the DL SCH-PDCCH channel, judged as downlink transfer message, subject to the respective sub-layer processing.

3) Sample three:

0002003c07b500010000000000290000003d00003c20191f50300000001660129b2e6016a4bfd56 $7 \mathrm{e} 283209 \mathrm{c} 00022 \mathrm{f} 1 \mathrm{~b} 3042500 \mathrm{a} 000400000000000000000000$

Analytical results from underlying interface:

3c20191f50300000001660129b2e6016a4bfd567e283209c00022flb3042500a000400000000000 000

Analytical results in MAC layer:

60129b2e6016a4bfd567e283209c00022flb3042500a000400

Manually verify analytical results are correct, the message is carried by the DLSCH-BCCH channel. After parsing from the underlying interface and the MAC layer processing, then delivers directly to RRC layer processing, analysis results find it RRC connection setup message.

4) Sample four: the size of message packet is big, divided into two frames

000f005458fe2b9f058e000104fd010500065d8f0000104b00000035010000003d3a010e1b880000 202000070b3a0741710bf664f02000070bc000e81 f02c0f0001d0201d0112717808021100101001081 $0600000000113 b$

000f0078599091550597000104fd0101000497fc00000bc200000059010000003d21191f00b0010 0830600000000000a005264f02001025c0493000000004341434141410000200001c2c2b60f0a04b6 0fffff00890089003a6facd $1250110000100000000000020454 a 464445424645454246414341434143$ 064450

Analytical results in MAC layer:

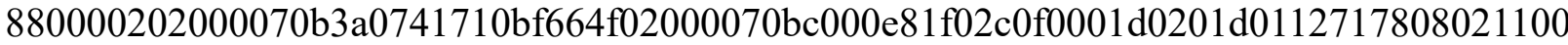
10100108106000000

Analytical results in RLC layer:

00202000070b3a0741710bf664f02000070bc000e81f02c0f0001d0201d01127178080211001010 0108106000000

The first segment

Analytical results in MAC layer:

b00100830600000000000a005264f02001025c049300000000

Analytical results in RLC layer:

$00830600000000000 \mathrm{a} 005264 \mathrm{f} 02001025 \mathrm{c} 049300000000$

The above parsed message belongs to the last segment ,the first segment with the last segment makes up a PDCP PDU results:

00202000070b3a0741710bf664f02000070bc000e81f02c0f0001d0201d01127178080211001010 $010810600000000830600000000000 \mathrm{a} 005264 \mathrm{f} 02001025 \mathrm{c} 049300000000$

Analytical results in RRC layer:

\{

message c1: rrcConnectionSetupComplete: \{

rrc-TransactionIdentifier 0 ,

criticalExtensions c1: rrcConnectionSetupComplete-r8: \{

selectedPLMN-Identity 1 ,

registeredMME \{

mmegi '0000000000000111'B,

mmec '00001011'B

\},

dedicatedInfoNAS

'0741710BF664F02000070BC000E81F02C0F0001D0201D01127178080211001010010810600000 
\}

\}

The message is carried by the DLSCH-DCCH channel, has the need for stripping header processing each sub-layer. The size of message packet is big, transmitted into two frames, obviously, the receiver must splice the two frames. The analysis results in RRC-layer know that the message is RRC connection setup complete.

\section{Conclusion}

Considering the characteristics of GEO satellite communication, here number of bits of SN for RLC AM PDU is extended to 18 , and the effective bits are 12bits in this paper. Designed protocol stack will be used in GEO satellite communication system, with further analysis of the protocol. Highlighting protocol analysis software architecture and the difficulties of protocol analysis, the paper designs bottom-up protocol analysis software framework based on different channel, and analyzes the problem of collage of data package in it. Finally, it is tested in a lab environment. The test results show the correctness of the design for protocol analysis software, which is able to track the dynamic process of the user, such as the state of attachment and detachment can be observed in protocol analysis platform.

\section{Acknowledgement}

The work described in this paper is supported by the project of National "863" Project of China No.2012AA01A502, No.2012AA01A505.

\section{References}

[1] Luan Xi, Xu Xiaoyan, Xiang Haige, etc. Adaptability Research on LTE air interface in a satellite communication system [C] // sixth satellite communication technology of new business Annual Conference Proceedings, 2010: 77-91.

[2] Chen Changmeng, Ceng Weijun.The Adaption of LTE Interface in Satellite Communication[J]. Wireless Communication Technology,2011,20(4):20-23.

[3] Yi Kechu, Li Yi, Sun Chenhua, etc. Recent development and its prospect of satellite communications[J] Journal of Communication, 2015, 36(6):157-172.

[4] Dou Zhibin.Study on Applicability of LTE RLC UM Mode in Mobile Satellite Communications[J]. Radio Communications Technology, 2014, 40(6):6-8.

[5] 3rd Generation Partnership Project Technical Specification Group Radio Access Network. Evolved Universal Terrestrial Radio Access (E-UTRA) Radio Link Control (RLC) protocol specification (Release 9) 3GPP TS 36.322 V9.3.0[S]. 2012.

[6] Huang Xiaoying, Cheng Yuxin, Wu Jianjun. A protocol stack software architecture solution for mobile satellite communication terminal [C] // seventh satellite communication new technologies, new business Annual Conference Proceedings 2011.

[7] 3rd Generation Partnership Project Technical Specification Group Radio Access Network. Evolved Universal Terrestrial Radio Access (E-UTRA) Radio Resource Control (RRC) Protocol Specification (Release 9) 3GPP TS 36.331 V9.3.0[S]. 